\title{
EXPERIÊNCIA DO USUÁRIO EM UM DOS APLICATIVOS DO GOOGLE: ANÁLISE DO INBOX
}

\author{
Bruna Ciafrei Rinaldi (1) \\ Fausto Orsi Medola (2) \\ Luis Carlos Paschoarelli (3) \\ (1) IESB-PREVE, Bacharel em Design \\ E-mail: contato@brunaciafreidesigner.com.br \\ (2) PPG-Design-Unesp, Doutor em Bioengenharia \\ E-mail: fausto.medola@faac.unesp.com.br \\ (3) PPG-Design-Unesp, Livre-Docente em Design \\ E-mail: paschoarelli@faac.unesp.com.br
}

\begin{abstract}
RESUMO
O Inbox é um aplicativo do Google desenvolvimento para facilitar a realização de tarefas em sua caixa de entrada de e-mails. O objetivo deste estudo foi analisar o usuário na realização de três tarefas aplicadas e coletar dados para testar a usabilidade da plataforma em termos de eficiência, satisfação, dificuldade e tempo médio estimado. Os resultados apontam que a interface, que conta com o visual do Material Design (nova guideline do Google), é apta e facilita ações dos usuários rapidamente. Esse estudo contribuiu para a análise dos métodos de aplicações de tarefas em sistemas digitais, para que usuários alcance seus objetivos em um curto tempo, com auxílio da boa usabilidade dessas interfaces.
\end{abstract}

\begin{abstract}
Inbox is a Google application development to facilitate the completion of tasks in your email inbox. The aim of this study has been to analyze the user in three applied tasks and collect data to test the platform's usability in terms of efficiency, satisfaction, difficulty and estimated average time. The results show that the interface that, which the look of Material Design (new guideline Google), is suitable and facilitates user actions quickly. This study has contributed to the analysis of the tasks of application methods in digital systems, so that users reach their goals in a short time with the aid of good usability of these interfaces.
\end{abstract}




\section{INTRODUÇÃO}

Com a crescente modernização das interfaces de web e os novos sistemas aplicados em plataformas digitais, a interação com os usuários se tornou o principal objeto de análise do UX (user experience). O UX visa estudar o ambiente interativo do usuário e averiguar os caminhos, as coordenadas e as conclusões que o mesmo percorre. A arquitetura de informação aplicada nos websites está agregando valor e gerando resultados positivos. $E$ isto decorre na otimização e gerenciamento de interfaces com maior qualidade e rapidez.

O UX está associado à Ergonomia, uma vez que foca no atendimento das necessidades dos usuários, como eles se comportam nos ambientes, o que interfere em sua relação com o cotidiano e, respectivamente, com a interface que o mesmo escolhe para se relacionar.

Uma das maiores empresas multinacionais que analisa a relação do usuário com o produto é o Google, a qual possui diversos serviços inclusos no mercado digital. No final de 2014, com a entrada do Android 5.0 (Lollipop) - um de seus produtos, foi lançado um guideline, e/ou framework, que apresenta uma unificação das interfaces obtidas pelo Google, visando melhor representar os recursos oferecidos: o denominado Material Design.

Entretanto, com essa inovação surgem algumas questões: Será que esse sistema proporciona ao usuário uma perspectiva ergonômica suficiente para se obter bons resultados, do ponto de vista da interação? Esta questão será analisada no presente estudo, juntamente com a análise dos recursos e dos usuários dentro de um dos aplicativos escolhidos para estudo, o Inbox.

\section{FUNDAMENTAÇÃO TEÓRICA}

\subsection{A usabilidade de interfaces}

Usabilidade é o fator que assegura, na perspectiva do usuário, a utilização dos produtos de forma fácil, eficiente e agradável (Preece et al., 2007). Dul e Weerdmeester (2004) afirmam que um usuário aciona o controle sobre uma determinada expectativa, e caso seu efeito não ocorra, podem acontecer problemas.

Para Memória (2003), o usuário pouparia tempo se as interfaces tivessem elementos mais padronizados, pois facilitariam a utilização e o aprendizado. A explicação disto é suscitada a partir da observação de Montmollin (1990): os usuários utilizam mais a indução do que a lógica dedutiva durante uma operação.

Para otimizar os sistemas interativos, Preece et al. (2007) propõe metas de usabilidade, assim como: (1) eficácia, cumpre o que o usuário espera do sistema; (2) eficiência, auxilia os usuários na realização de suas tarefas; (3) segurança, protege e evita situações perigosas e indesejáveis; (4) utilidade, oferece funcionalidades que dão sentido à existência do sistema; (5) aprendizagem, configura-se de forma a ser o mais facilmente assimilada e (6) memorização, funciona de forma lógica e capaz de ser lembrada.

A usability engineering ganhou destaque no cenário mundial de pesquisa e desenvolvimento de interfaces, por meio das propostas de Nielsen (1993). De fato, a usabilidade de interfaces deveria ser avaliada e desenvolvida com base em critérios ergonômicos rígidos e da forma mais simples possível, elaborada com elementos estritamente funcionais e indispensáveis. Esses critérios, conhecidos como "Heurística de Nielsen", são os seguintes: (1) visibilidade do status do sistema; (2) equivalência entre o sistema e o mundo real; (3) controle do usuário e liberdade; (4) consistência e padrões; (5) prevenção de erros; (6) reconhecimento ao invés de recordação; (7) flexibilidade e eficiência de uso; (8) estética e design minimalista; (9) apoio aos usuários a reconhecer, diagnosticar e a recuperar ações erradas e (10) ajuda e documentação. 


\subsection{O contexto da engenharia de usabilidade}

Dentro do contexto de Engenharia de Usabilidade, Nielsen fora contratado em 1994 como distinguished engineer pela Sun Microsystems, com a missão de facilitar o uso de softwares coorporativos da empresa. O que aconteceu é que, nesse mesmo período, a World Wide Web $^{9}$ tornava-se um sucesso comercial e a web usability (em tradução livre, usabilidade para rede) ganhava força, alcançando Nielsen o status de guru mundial da usabilidade - apelido que Ihe foi atribuído pelo jornal norte-americano The New York Times. Naquela época, diversos desenvolvedores para web, principalmente no lado do design visual, criticaram a proposta de Nielsen para avaliar a usabilidade. A principal crítica centrava-se principalmente no fato de a engenharia da usabilidade ser fundada em uma visão simplista, quase maniqueísta, do que seria um "bom design" e um "mau design" (JARDIM e OLIVEIRA, 2015).

Segundo a ISO, usabilidade por ser caracterizada como a "medida em que um sistema, produto ou serviço pode ser usado por usuários específicos para atingir objetivos específicos com eficácia, eficiência e satisfação em um determinado contexto de uso". (ISO, 2010, p.5).

A usabilidade considera o usuário como peça essencial no processo de interação. A experiência de usuários específicos, utilizando sistemas para finalidades específicas, torna-se mais efetiva, eficiente e satisfatória. Jardim e Oliveira (2015) afirmam ainda que a usabilidade diz respeito à adequação de um sistema às necessidades dos usuários, permitindo verificar o seu desempenho e conhecer a satisfação dos mesmos ao realizar determinadas tarefas.

Nielsen (2012), enumera cinco atributos passíveis de mensuração relacionados à usabilidade: (1) facilidade de aprendizado; (2) eficiência de uso; (3) facilidade de memorização; (4) baixa taxa de erros e (5) satisfação subjetiva. A avaliação heurística, segundo Nielsen e Molich apud. Moreira, 2007, é um método onde "[...] um avaliador procura problemas de usabilidade em uma interface através de análises e interpretações de um conjunto de princípios ou heurísticas". Santos et al. (apud Preece et al., 2008) mencionam que a avaliação heurística desenvolvida por Nielsen, constituise em uma técnica de inspeção de usabilidade em que especialistas, orientados por um conjunto de princípios, conhecidos como heurística, avaliam se os elementos de interface com o usuário - caixas de diálogos, menus, estruturas de navegação, ajuda on-line, entre outros - estão de acordo com os princípios.

\subsection{UX - User Experience}

A experiência permeia a vida humana em todos os aspectos. Para Oliveira, et al. (apud Dewey, 1980), uma experiência constitui-se fruto da interação de pessoas com o contexto em que vivem. Nesse sentido, o fenômeno da experiência sempre existiu, e segundo Ferreira (2011), está intimamente ligado aos cinco sentidos humanos, que por sua vez, interagem com a cognição do sujeito em atividades cotidianas.

Já o User Experience - experiência do usuário, ou UX - conforme defende Franz (2015, apud Nielsen 2008), não só inclui a usabilidade, mas também os aspectos cognitivos, socioculturais e afetivos - aspectos positivos de experiência dos usuários em sua interação com os produtos, além da experiência estética ou desejo de reutilizá-los.

Para Araújo (2014), a usabilidade associa-se ao prazer que o usuário tem em realizar tarefas que estão relacionadas com as percepções estéticas e emocionais.

No âmbito do design, o foco na experiência começa a figurar de forma mais contundente em projetos realizados a partir dos ano 90, com a emergência da "economia da experiência" (McLellan, 2000). Nos períodos anteriores, as abordagens refletiam produtos ou serviços primordialmente voltados para as suas características estruturais (forma e função) como, por exemplo, os relógios, que durante muito tempo, foram tratados como objetos limitados a sua função de uso (Oliveira, 2014 et al., apud Cardoso 2012). 
Para Oliveira et al. (apud Hartson e Pardha, 2012), a experiência do usuário é a totalidade do efeito, ou efeitos sentidos internamente por usuários, como resultado da integração com o ambiente ou contexto de uso. Além dos fatores humanos internos, a experiência do usuário é influenciada por outros elementos externos, tais como as pessoas e os objetos como os quais um indivíduo pode interagir através dos seus sentidos, bem como as atividades realizadas nesse contexto.

\subsection{Projetando experiência para o usuário}

Projetar produtos interativos usáveis, segundo Oliveira et al. (2014, apud Preece et al. 2005), requer que seja levado em conta por quem serão utilizados e o seu contexto de uso. Outra preocupação importante consiste em entender o tipo de atividade que os usuários realizam, quando estão interagindo com os produtos.

Desse modo, em um projeto para experiência do usuário, é preciso considerar um conjunto de elementos: para quem será destinado o projeto (é necessário conhecer especificidades correspondentes ao usuário que irá participar da experiência); o que será projetado como objeto da experiência (se é um produto, um serviço, uma interface, ou uma nova tecnologia); o objetivo da interação (por exemplo, criar uma experiência de interação imersiva por meio de uma interface atraente e fácil de operar); onde a experiência ocorrerá (seu contexto específico de uso, seja físico, social, tecnológico, etc.); e quando a experiência ocorrerá (considerando que, de acordo com aspectos cognitivos e emocionais do usuário, a experiência pode iniciar antes mesmo da interação).

Segundo Bonsiepe (2012), a preocupação central do design está justamente na otimização da relação usuário e produto, contribuindo para o processo de desenvolvimento do produto de forma integrada, em que a aparência do produto não deve ser interpretada de forma especial, como algo agregado, mas sim conformada com a estrutura de solução. Deste modo, por meio da aparência, o designer busca imprimir personalidade ao produto, estimulando as emoções e comportamento dos usuários.

Embora não sejam previsíveis nem controláveis por parte do designer, as emoções podem ser estudadas a fim de conhecer o seu impacto no comportamento dos usuários. Sendo assim, de acordo com Oliveira et al. (2014), é importante esclarecer um aspecto essencial para a compreensão do projeto de design de interação: não é possível projetar emoções em um produto. Portanto, o autor afirma que não se pode "projetar a experiência do usuário", mas sim "projetar para a experiência do usuário".

\subsection{Google e o Material Design}

No final de 2014, foi o lançado o Material Design, uma das grandes novidades do Google I/O, a nova guideline (guia-padrão) ou framework (em tradução livre guia para desenvolvedores) de design do Google, que promove um layout baseado no conceito de profundidade e 3D. Mas não é só aí que está a inovação.

O diferencial deste design é a funcionalidade e a preocupação com o usuário. Já ficou claro que não adianta algo ser bonito e não ser funcional, ou vice-versa, e o Google conseguiu encontrar esse equilíbrio e unificar a experiência do usuário em suas variadas plataformas. Dentro dessa guideline foram definidos alguns princípios que otimizam a usabilidade desse material como: (1) o formato é uma linha tênue entre beleza e "breguice", que funciona. A ideia, apresentada pelo vice-presidente de Design do Google Matias Duarte, é criar experiências parecidas com as que temos com objetos reais. Este é o primeiro princípio da guideline, mostrado no próprio site de introdução, "material is the metaphor".

\subsection{O UX e o Material Design}

Os designers são conhecidos por muitas funções: designer de produtos (físicos), designer de imagens, designer de interfaces web, designer gráfico, designer mobile, 
etc. Nos últimos anos surgiu uma nova carreira: UX (user experience), que muitos ainda confundem com UI (user interface). De fato as duas coisas são interligadas, porém, um não pode ser feito sem o outro e cada um tem seu próprio mérito.

O trabalho do UX (profissional de user experience) é entender, criar e descrever o melhor fluxo para as coisas funcionarem e, em geral, nas empresas que já possuem essa estrutura. Por sua vez, o responsável por UI (profissional de user interface) recebe os wireframes ou mockups desenhados pelo UXer para trabalhar a partir daí.

Por meio do Material Design, o Google junta o melhor de dois mundos, fazendo com que seja necessário pensar em formas de fazer um design bonito funcionar em um fluxo objetivo e simples. Uma mudança que já era bastante necessária e que impacta a forma como todos os designers devem trabalhar. O futuro do design está não só na beleza estética, mas também no aspecto dinâmico (CAMPOS, 2014).

Além disso, o lançamento do Material Design veio acompanhado do 'firmware' para o Android 5.0 - Lollipop que trouxe muitas inovações nos conceitos gráficos e funcionais.

Alguns aplicativos já utilizam o Material Design como o guia de direcionamento para a construção das plataformas, são eles: (1) Inbox by Gmail; (2) Field Trip; (3) Wealther Timeline; (4) SwiftKey; (5) Action Launcher 3; (6) Falcon Pro 3; (7) Simplenote; (8) Messenger from Google; (9) QKSMS; (10) Cabinet Beta; (11) Numix Calculator Base; (12) Chrooma Live Wallpaper; (13) Source News Reader; (14) Nova Launcher (beta); (15) RunKeeper; (16) Truedialer; (17) OneCalc; (18) Material Manager; (19) Wave Calendar; (20) Voltage Music Player.

\subsection{O aplicativo INBOX}

O Google impressionou o mundo ao anunciar o Inbox, que pode ser considerado o sucessor do clássico Gmail. Trata-se de um aplicativo de e-mails que trabalha com um formato diferenciado, permitindo que você tenha maior controle das mensagens que chegam na sua caixa de entrada.

Segundo Souza (2016), um dos primeiros aplicativos a ser construído com base no Material Design, o Inbox é composto por clientes para Android e iOS e uma interface que pode ser acessada a partir de qualquer navegador. Para acessar o Inbox, é necessário requisitar um convite da própria Google ou de algum amigo que já esteja utilizando o novo serviço. O aplicativo contém alguns recursos que ajudam a facilitar a integração dos e-mails dos usuários,ajudando-os a melhorar atividades, tarefas, lembretes e notas recebidas ou enviadas.

O Inbox possui dentro da sua plataforma alguns recursos na barra lateral, segmentados como: Entrada, Adiados, Concluídos, Rascunhos, Enviados, Lembretes, Lixeira, Spam e Contatos, além de agrupar alguns marcadores relacionados a: Viagens, Compras e Finanças, e também disponibiliza as seções personalizadas. Além desses recursos alguns marcadores fixos também se relacionam com a interface inicial, como: Social, Atualizações, Fóruns e Promoções.

A linha do tempo, onde os e-mails ficam marcados, possui ícones que ajudam a facilitar o desempenho da tarefa a ser tomada como: Marcar na Entrada, Adiar até..., Marcar como Concluído e Mover para... Algumas opções icônicas também ficam no topo da interface que simbolizam alguns recursos a serem tomados pelos seus usuários. 
Figura 1: Caixa de Entrada do Inbox (tela inicial)

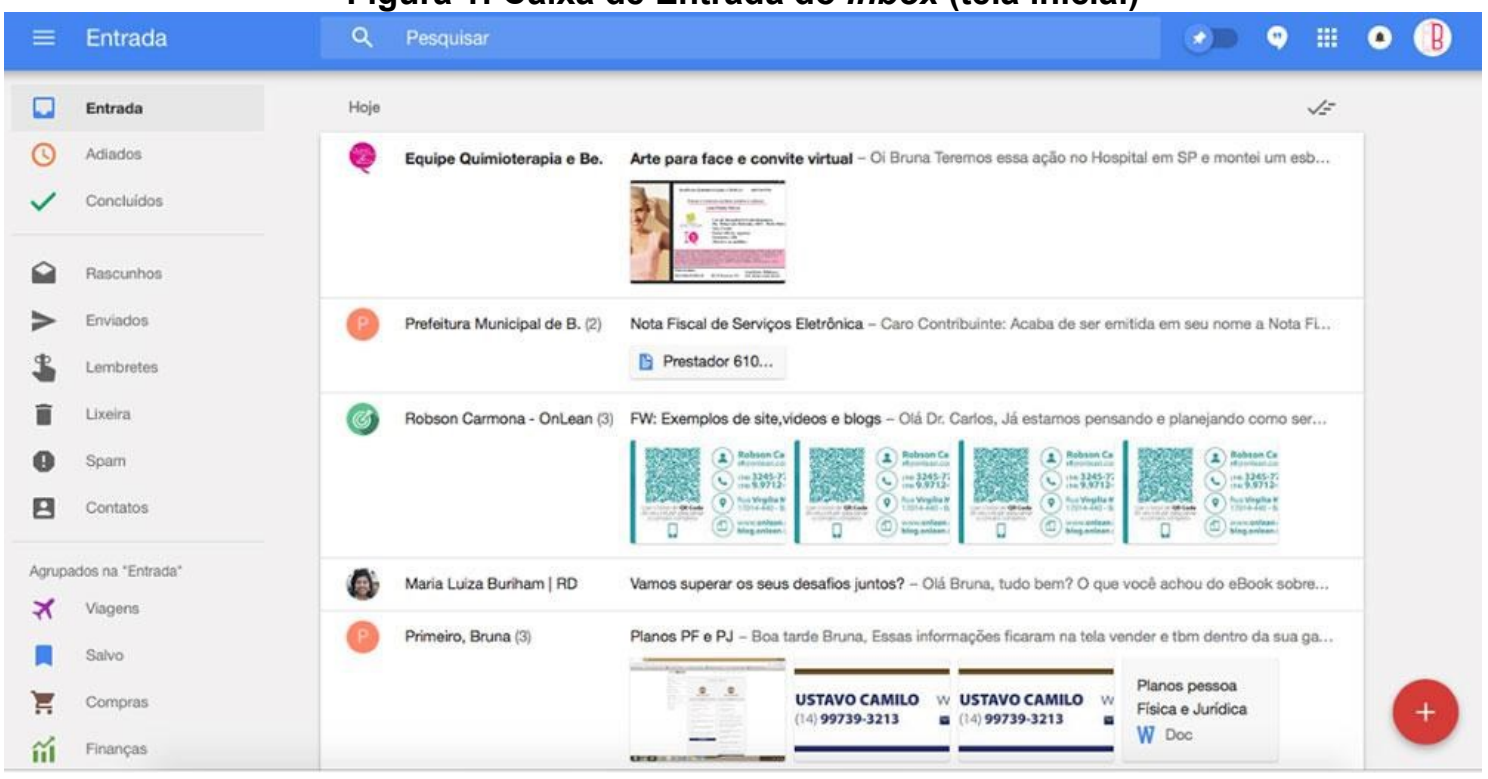

\section{OBJETIVOS}

O objetivo deste estudo foi analisar o usuário na realização de três tarefas aplicadas dentro do aplicativo Inbox. O propósito foi abordar a usabilidade da ferramenta para que os usuários pudessem interagir de forma rápida e prática nesse novo modelo de e-mail desenvolvido pelo Google. Além disso, o teste contou com um método de avaliação aplicado para os níveis de satisfação, insatisfação, eficácia, ineficácia, grau de dificuldade e tempo médio estimado.

\section{MATERIAIS E MÉTODOS}

O presente estudo caracteriza-se como transversal e experimental. A participação de sujeitos em atividades simuladas de uso do aplicativo Inbox foi possível com a concordância dos mesmos e o preenchimento e assinatura de um Termo de Consentimento Livre e Esclarecido.

\subsection{Amostragem}

Participaram do estudo seis usuários, sendo três homens e três mulheres, com idade média 25 a 40 anos. Todos os participantes relataram utilizar internet e as interfaces do Google.

\subsection{Materiais}

Para a realização das tarefas foram utilizados computadores e/ou notebooks em um laboratório de informática. Além desses instrumentos, foi utilizado um cronômetro para medir o tempo médio e uma formulário impresso com a seguinte tabela de dados:

- Tempo de execução das tarefas

- Grau de dificuldade

- Eficácia da plataforma

- Grau de satisfação 


\subsection{Métodos}

Para avaliar o nível de interação e tempo da interface, foi aplicada uma escala de 1 a 10 para os tais: tempo, dificuldade, eficácia e satisfação:

- Tempo de execução das tarefas: tempo ilimitado

- Grau de dificuldade: sendo 0 para fácil e 10 para difícil

- Eficácia da plataforma: sendo 0 para ineficaz e 10 para eficaz

- Grau de satisfação: sendo 0 para insatisfeitos e 10 para satisfeitos

\subsection{Procedimentos}

Testes de usabilidade são termos usados frequentemente para referir-se a quaisquer técnicas de avaliação de um produto ou sistema. No entanto, de acordo com Franz et al. (2015, apud Rubin, 2008) o termo "teste de usabilidade" refere-se a um processo que emprega pessoas como participantes de testes que representam o público-alvo e avaliam o grau em que um produto atendeu a usabilidade de tal ferramenta.

Franz et al. (2015, apud Cybis, 2007), afirma que a avaliação feita em um laboratório equipado com dispositivos e aparelhos de registro, confere maior poder de controle e de observação da interação do usuário com o produto. O responsável pela avaliação pode modificar a sequência ou introduzir novas tarefas, além de definir a melhor posição para que as pessoas observem a interface aplicada.

A especificação listada no objetivo das realizações das tarefas, dos materiais e da metodologia foi a aplicação do método de avaliação do aplicativo Inbox. Essas tarefas foram caracterizadas em 3 etapas:

1. Conectar o Gmail com o aplicativo Inbox e interagir com alguns e-mails dados como não lidos e tentar fazer quaisquer resoluções das atividades para destinar esses e-mails em: Entrada, Adiados e Concluídos.

2. Criar um e-mail para qualquer usuário de sua preferência e enviar o mesmo.

3. Criar um lembrete qualquer e depois ir até a tela de lembretes para interagir com o mesmo, dando a ele alguma marcação.

Não foram utilizados dispositivos móveis para esse trabalho específico, pois o objetivo seria tratar a plataforma em um ambiente interno (laboratório) e avaliar a postura das amostras na versão desktop. A análise de dados caracterizou-se como descritiva.

\section{RESULTADOS}

\subsection{Tempo de execução da tarefa}

O tempo de execução das tarefas apresentaram elevada variabilidade entre os diferentes participantes (Tabela 1).

Tabela 1: Tempo de Execução das três tarefa (em segundos (')).

\begin{tabular}{|l|l|}
\hline No de participante & Tempo em (') \\
\hline Participante 1 & 1 ' \\
\hline Participante 2 & 2 ' \\
\hline
\end{tabular}




\begin{tabular}{|l|l|}
\hline Participante 3 & $10^{\prime}$ \\
\hline Participante 4 & $1^{\prime}$ \\
\hline Participante 5 & $4^{\prime}$ \\
\hline Participante 6 & $2^{\prime}$ \\
\hline Média & $3,5^{\prime}$ \\
\hline
\end{tabular}

\subsection{Grau de dificuldade das tarefas}

O grau de dificuldade das tarefas apresentaram baixa variabilidade entre os diferentes participantes (Tabela 2).

Tabela 2: Grau de dificuldade (avaliação dos usuários)

\begin{tabular}{|l|l|}
\hline No de participante & De $\mathbf{0}$ a 10 \\
\hline Participante 1 & 2 \\
\hline Participante 2 & 1 \\
\hline Participante 3 & 0 \\
\hline Participante 4 & 2 \\
\hline Participante 5 & 4 \\
\hline Participante 6 & 1 \\
\hline Média & $\mathbf{1 , 6}$ \\
\hline
\end{tabular}

\subsection{Eficácia da plataforma}

A eficácia das tarefas apresentaram média variabilidade entre os diferentes participantes (Tabela 3).

Tabela 3: Eficácia da plataforma (relação da usabilidade) 


\begin{tabular}{|l|l|}
\hline No de participante & De 0 a 10 \\
\hline Participante 1 & 8 \\
\hline Participante 2 & 10 \\
\hline Participante 3 & 5 \\
\hline Participante 4 & 9 \\
\hline Participante 5 & 7 \\
\hline Participante 6 & 10 \\
\hline Média & $\mathbf{8 , 2}$ \\
\hline
\end{tabular}

\subsection{Grau de satisfação}

O grau de satisfação das tarefas apresentaram baixa variabilidade entre os diferentes participantes (Tabela 3).

Tabela 4: Grau de satisfação (praticidade e rapidez)

\begin{tabular}{|l|l|}
\hline No de participante & De 0 a 10 \\
\hline Participante 1 & 8 \\
\hline Participante 2 & 10 \\
\hline Participante 3 & 6 \\
\hline Participante 4 & 9 \\
\hline Participante 5 & 8 \\
\hline Participante 6 & 10 \\
\hline
\end{tabular}




\begin{tabular}{|l|l|}
\hline Média & 8,5 \\
\hline
\end{tabular}

\section{DISCUSSÕES E CONSIDERAÇÕES FINAIS}

De acordo com os resultados alcançados no presente estudo durante a coleta paramétrica, avaliou-se o nível de satisfação perante os resultados obtidos dos usuários selecionados para esse procedimento. Isso sugere que o Material Design possa ser uma plataforma com um design aplicável em outras superfícies digitais por proporcionar eficácia no seu manuseio e proporcionar uma experiência útil para os usuários.

Este estudo contribuiu para as abordagens aos métodos de avaliação de atividades em sistemas digitais, que ergonomicamente ajudariam o usuário a chegar em seu objetivo em um curto tempo com auxílio da boa usabilidade de tais interfaces.

O Inbox já é um sistema apreciado pela integração e a facilidade da realização de tarefas recebidas por e-mail. Além disso, ajuda o usuário a determinar suas ações para melhor realizar agendamentos, notificações e descarte dos e-mails recebidos, além de interagir com lembretes, notas e organização das tarefas recebidas.

A tendência do Google, é unificar suas plataformas e aplicar esse estilo de design para otimizar a prática de suas atividades e melhorar o desempenho e uso dos sistemas operacionais.

\section{REFERÊNCIAS BIBLIOGRÁFICAS}

ARAUJO, F. S. Avaliação da experiência do usuário: uma proposta de sistematização para o processo de desenvolvimento de produtos. UFSC, 2014.

BONSIEPE, G. Design - the blind spot of theory of virtuality | Discursivity of Theory - the blind spot of design. Disponivel em </www.guibonsiepe.com/pdffiles/visudisc.pdf>. Acesso em 6 junho de 2016.

Design como prática de projeto. São Paulo: Blucher, 2012.

CAMPOS, M. Como o Google Material vai mudar o trabalho dos designers. Disponível em <http://blog.concretesolutions.com.br/2014/12/google-materialdesigners/> Acesso em 10 maio de 2016.

CARDOSO, R. Design para um mundo complexo. São Paulo: Cosac Naify. 2012.

CYBIS, W. Ergonomia e usabilidade: conhecimentos, métodos e aplicações. São Paulo; Novatec Editora, 2007.

DEWEY, J. Experiência e natureza; Lógica: a teoria da investigação; A arte como experiência; Vida e educação; Teoria da vida moral. São Paulo: Abril Cultura, 1980.

DUL, J; WEERDMEESTER, B. Ergonomia prática. 2.ed. São Paulo: Edgard Blucher, 2004.

FERREIRA, N. G. M. L. O papel da experiência na filosofia de John Dewey.

Filogênese, São Paulo,. v.4, n. 2, p. 147-156, 2011. 
FRANZ, A. M. S., SANTOS, F. A. N. V. Métodos para análise da experiência do usuário em laboratório, p. 906-916 . In: Anais do $15^{\circ}$ Ergodesign \& Usihc. Blucher Design Proceedings, vol. 2, n. 1. São Paulo: Blucher, 2015. ISSN 2318-6968, DOI 10.5151/15ergodesign-53-E115

GOOGLE (․ Documentation of Material Design: guideline. Disponível em $<$ https://www.google.com/design/spec/material-design/introduction.html> Acesso em 10 maio de 2016.

HARTSON, R \& PARDHA, P. S. The UX book. Process and guidelines for ensuring a quality user experience. Waltham: Elsevier, 2012.

JARDIM, A. J. F. \& OLIVEIRA, S. R. R. Paradigmas de critérios ergonômicos para avaliação de interfaces web: um paradoxo? E-Tech: Tecnologias para Competitividade Industrial, Florianópolis, n. Especial Design. 2015/1.

MCLELLAN, H. Experience design. Cyberpycology \& Behavior, v. 3, n. 1. p.59-69, 2000.

MEMORIA, F. Usabilidade de interfaces e arquitetura de informação. Navegação Estrutural. $2^{\circ}$ Congresso Internacional de Ergonomia e Usabilidade, Design de Interfaces e Interação Homem-Computador - USIHC. Rio de Janeiro, 2003.

NIELSEN, J \& MOLICH, R. Heuristic evaluation of user interfaces. In Proceedings of the SIGCHI Conference on Human Factors in Computiing Systems, New York: ACM, 1990.

Academic, 2008 .

Designing web usability; the practise of simplicity. Boston, USA;

Designing web usability: the practise of simplicity. New Riders Publishing, Indianapolis, 1999.

Usability 101: Introduction to usability. Freemont: Nielsen Norman Group, 2012. Disponível em <www.nngroup.com/articles/usability-101-introduction-tousability/> Acesso em 5 junho de 2016.

Usability Engineering. San Diego: Academic Press, 1993.

OLIVEIRA, R. N.; LIMEIRA, C. D.; ROSA, J. G. S.; A experiência do usuário no processo evolutivo do design, p. 3451-3460 . In: Anais do $11^{\circ}$ Congresso Brasileiro de Pesquisa e Desenvolvimento em Design. Blucher Design Proceedings, v. 1, n. 4. São Paulo: Blucher, 2014 | ISSN 2318-6968, DOI 0.5151/designpro-ped-01353

PREECE, J., ROGERS, Y. \& SHARP, H. Design de interação: além da interação homem-computador. Porto Alegre: Bookman, 2008.

SANTOS, D. B., MOURA, A. M. M. \& PAVÃO, C. M. G. Proposta metodológica para avaliação de usabilidade de interfaces de repositórios. Cadernos $B A D, 2014$. n. 2 , jul-dez, pp.215-218.

SOUZA, Ramon de. Inbox: saiba como usar o novo serviço do Google. Disponível em: <http://www.tecmundo.com.br/inbox/65457-inbox-saiba-usar-novo-servicogoogle.htm> Acesso em 5 de junho de 2016. 